\title{
Mixed Venous Oxygen Tension
}

National Cancer Institute

\section{Source}

National Cancer Institute. Mixed Venous Oxygen Tension. NCI Thesaurus. Code C129960.

The partial pressure of oxygen in venous blood, representing the balance between oxygen consumption and oxygen delivery. 